\title{
A Quasi-Hopf algebra interpretation of quantum 3-j and $6-j$ symbols and difference equations.
}
O. Babelon ${ }^{\dagger}$
D. Bernard *
E. Billey ${ }^{\dagger}$

\begin{abstract}
We consider the universal solution of the Gervais-Neveu-Felder equation in the $\mathcal{U}_{q}\left(s l_{2}\right)$ case. We show that it has a quasi-Hopf algebra interpretation. We also recall its relation to quantum $3-\mathrm{j}$ and 6 -j symbols. Finally, we use this solution to build a q-deformation of the trigonometric Lamé equation.
\end{abstract}

PAR LPTHE 95-51, IHES/P/95/91

\footnotetext{
*Member of CNRS, Service de Physique Théorique, CEN-Saclay, Orme des Merisiers, F-91191, Gif-surYvette, France and IHES 35 route de Chartres, 91440 Bures sur Yvette, France.

${ }^{\dagger}$ L.P.T.H.E. Université Paris VI (CNRS UA 280), Box 126, Tour 16, $1^{\text {er }}$ étage, 4 place Jussieu, 75252 Paris Cedex 05, France
} 


\section{Introduction}

The Gervais-Neveu-Felder equation is a deformation of the standard Yang-Baxter equation. In the $s l_{2}$ case, it reads

$$
R_{12}(x) R_{13}\left(x q^{H_{2}}\right) R_{23}(x)=R_{23}\left(x q^{H_{1}}\right) R_{13}(x) R_{12}\left(x q^{H_{3}}\right)
$$

Here, $H$ denotes a Cartan generator in $s l_{2}$ (or rather $\mathcal{U}_{q}\left(s l_{2}\right)$ ) and $x$ is a parameter not to be confused with the spectral parameter (absent in the $s l_{2}$ case).

This equation appeared independently in several contexts. It was first discovered by J.L. Gervais and A. Neveu in their studies on Liouville theory [1]. It was rediscovered by G. Felder in his approach to the quantization of the Knizhnik-Zamolodchikov-Bernard equation [2]. Finally, it was shown to play an important role in the quantization of the Calogero-Moser models in the $R$-matrix formalism [3]. For all these reasons, we believe that this equation deserves much attention.

In this note, we analyse the universal solution $R(x) \in \mathcal{U}_{q}\left(s l_{2}\right) \otimes \mathcal{U}_{q}\left(s l_{2}\right)$ of eq.(1) obtained in [⿴囗才]. We show that it has a nice quasi-Hopf algebra interpretation. For completeness, we recall the connection of this solution with $q$-analogs of $3-\mathrm{j}$ and 6 -j symbols. Finally, we explain how it can be used to construct a $q$-difference analog of the trigonometric Lamé equation (Calogero model for 2 particules).

\section{A summary of universal formulae}

In this section, we recall the universal formulae obtained in $\mathbb{\|}$ for the matrix $R_{12}(x) \in$ $\mathcal{U}_{q}\left(s l_{2}\right) \otimes \mathcal{U}_{q}\left(s l_{2}\right)$. We denote by $H, E_{ \pm}$the generators of the quantum group $\mathcal{U}_{q}\left(s l_{2}\right)$

$$
\left[H, E_{ \pm}\right]= \pm 2 E_{ \pm}, \quad\left[E_{+}, E_{-}\right]=\frac{q^{H}-q^{-H}}{q-q^{-1}}
$$

The coproduct is defined as

$$
\Delta(H)=H \otimes i d+i d \otimes H, \quad \Delta\left(E_{ \pm}\right)=E_{ \pm} \otimes q^{\frac{1}{2} H}+q^{-\frac{1}{2} H} \otimes E_{ \pm}
$$

We have $R_{12}^{D} \Delta(a)=\Delta^{\prime}(a) R_{12}^{D}$ for any $a \in U_{q}\left(s l_{2}\right)$ where $\Delta^{\prime}$ is the opposite comultiplication and $R_{12}^{D}$ Drinfeld's universal $R$-matrix :

$$
R_{12}^{D}=q^{\frac{1}{2} H \otimes H} \sum_{i=0}^{\infty}\left(q-q^{-1}\right)^{i} \frac{q^{-\frac{i(i+1)}{2}}}{[i] !} q^{\frac{i}{2} H} E_{+}^{i} \otimes q^{-\frac{i}{2} H} E_{-}^{i}
$$

As usual, q-numbers are defined as $[i]=\left(q^{i}-q^{-i}\right) /\left(q-q^{-1}\right)$. Let us now define

$$
R_{12}(x)=F_{21}^{-1}(x) R_{12}^{D} F_{12}(x)
$$

with

$$
\begin{aligned}
F_{12}(x) & =\sum_{k=0}^{\infty}\left(q-q^{-1}\right)^{k} \frac{(-1)^{k}}{[k] !} \frac{x^{k}}{\prod_{\nu=k}^{2 k-1}\left(x q^{\nu} q^{H_{2}}-x^{-1} q^{-\nu} q^{-H_{2}}\right)} q^{\frac{k}{2}\left(H_{1}+H_{2}\right)} E_{+}^{k} \otimes E_{-}^{k} \\
F_{12}^{-1}(x) & =\sum_{k=0}^{\infty}\left(q-q^{-1}\right)^{k} \frac{1}{[k] !} \frac{x^{k}}{\prod_{\nu=1}^{k}\left(x q^{\nu} q^{H_{2}}-x^{-1} q^{-\nu} q^{-H_{2}}\right)} q^{\frac{k}{2}\left(H_{1}+H_{2}\right)} E_{+}^{k} \otimes E_{-}^{k}
\end{aligned}
$$


It follows from the construction of [1] that $R_{12}(x)$ is a solution of eq.(11).

One can check that $F_{12}(x)$ satisfies the following "shifted cocycle" condition

$$
[(i d \otimes \Delta) F] \cdot[i d \otimes F]=[(\Delta \otimes i d) F] \cdot\left[F\left(x q^{H_{3}}\right) \otimes i d\right]
$$

This relation is proved using standard q-binomial identities. It turns out that $F_{12}(x)$ is actually a "shifted coboundary"

$$
F_{12}(x)=\Delta M(x)[i d \otimes M(x)]^{-1}\left[M\left(x q^{H_{2}}\right) \otimes i d\right]^{-1}
$$

where the formula for the "boundary" reads

$$
M(x)=\sum_{n, m=0}^{\infty} \frac{(-1)^{m} x^{m} q^{\frac{1}{2} n(n-1)+m(n-m)}}{[n] ![m] ! \prod_{\nu=1}^{n}\left(x q^{\nu}-x^{-1} q^{-\nu}\right)} E_{+}^{n} E_{-}^{m} q^{\frac{1}{2}(n+m) H}
$$

Equation (5) implies eq. (4).

\section{Quasi-Hopf algebra interpretation}

The previous construction possesses a natural quasi-Hopf interpretation. Indeed, since $R(x)$ is defined in eq.(2) by a twisting procedure in the sense of Drinfeld [5] it is canonically associated to a quasi-Hopf structure on $U_{q}\left(s l_{2}\right)$. We shall denote it as $U_{q ; x}\left(s l_{2}\right)$. This quasiHopf algebra possesses very specific properties due to the "shifted cocycle" relation (4) satisfied by $F(x)$. Besides Drinfeld's construction, this gives another example of a quasiHopf algebra structure over $\mathcal{U}_{q}\left(s l_{2}\right)$.

Let us recall following ref. [5] that a quasi-Hopf algebra is specified by a quadruplet $(A, \Delta, R, \Phi)$ where $A$ is an assocative algebra, $\Delta$ is a (non-coassociative) comultiplication in $A, R \in A \otimes A$ and $\Phi \in A \otimes A \otimes A$ are such that :

$$
\begin{aligned}
R \Delta(a) & =\Delta^{\prime}(a) R \\
(i d \otimes \Delta) \Delta(a) \Phi & =\Phi(\Delta \otimes i d) \Delta(a)
\end{aligned}
$$

for all $a \in A$. There also are extra compatibility relations between $\Delta, R$ and $\Phi$ which we shall mention when needed. We will consider quasitriangular quasi-Hopf algebra, i.e., $R$ is assumed to verify the conditions

$$
\begin{aligned}
(\Delta \otimes i d) R & =\Phi_{321} R_{13} \Phi_{132}^{-1} R_{23} \Phi_{123} \\
(i d \otimes \Delta) R & =\Phi_{231}^{-1} R_{13} \Phi_{213} R_{12} \Phi_{123}^{-1}
\end{aligned}
$$

There exists a twisting procedure to construct quasi-Hopf algebras. Namely, if $(A, \Delta, R, \Phi)$ is a quasitriangular quasi-Hopf algebra then a new quasitriangular quasi-Hopf algebra $(A, \widetilde{\Delta}, \widetilde{R}, \widetilde{\Phi})$ is defined by $\widetilde{\Delta}(a)=F_{12}^{-1} \Delta(a) F_{12}$, and

$$
\begin{aligned}
\widetilde{\Phi} & =F_{23}^{-1}\left[(i d \otimes \Delta)\left(F^{-1}\right)\right] \Phi[(\Delta \otimes i d)(F)] F_{12} \\
\widetilde{R} & =F_{21}^{-1} R F_{12}
\end{aligned}
$$

with $F_{12} \in A \otimes A$. 
In our case, we are twisting $U_{q}\left(s l_{2}\right) \equiv\left(U_{q}\left(s l_{2}\right), \Delta, R^{D}, i d\right)$ by $F(x)$. So we have $\widetilde{R}=$ $F_{21}^{-1}(x) R_{12}^{D} F_{12}(x)=R(x)$ as defined in eq.(目). We denote $\widetilde{\Delta}$ by $\Delta_{x}$ with :

$$
\Delta_{x}(a)=F_{12}^{-1}(x) \Delta(a) F_{12}(x), \quad \forall a \in U_{q}\left(s l_{2}\right)
$$

It is a simple check to verify that the "shifted cocycle" condition (4) implies that :

$$
\left(i d \otimes \Delta_{x}\right) \Delta_{x}(a)=\left(\Delta_{x q^{H_{3}}} \otimes i d\right) \Delta_{x}(a)
$$

In other words, the shift breaks the co-associativity. We denote $\widetilde{\Phi}$ by $\Phi(x)$. It possesses a simple expression in terms of $F(x)$ :

$$
\begin{aligned}
\Phi(x) & =F_{23}^{-1}(x)\left[(i d \otimes \Delta)\left(F^{-1}(x)\right)\right][(\Delta \otimes i d)(F(x))] F_{12}(x) \\
& =F_{12}^{-1}\left(x q^{H_{3}}\right) F_{12}(x)
\end{aligned}
$$

where in the last equality we again used the "shifted cocycle" relation (4).

We can now write all the quasi-Hopf relations in $U_{q ; x}\left(s l_{2}\right)$ in terms of $R(x)$ or $F(x)$. For example, the general relation (7) reduces to eq.(11). Also, thanks to the following property,

$$
R_{12}\left(x q^{H_{3}}\right)=\Phi_{213}(x) R_{12}(x) \Phi_{123}^{-1}(x)
$$

the quasi- Yang-Baxter equation,

$$
\Phi_{321}^{-1}(x) R_{12}(x) \Phi_{312}(x) R_{13}(x) \Phi_{132}^{-1} R_{23}(x)=R_{23}(x) \Phi_{231}^{-1}(x) R_{13}(x) \Phi_{213}(x) R_{12}(x) \Phi_{123}^{-1}(x)
$$

valid in any quasitriangular quasi-Hopf algebra reduces to the equation (11).

Similarly, the quasitriangular property of $U_{q ; x}\left(s l_{2}\right)$ implies that

$$
\begin{aligned}
& \left(\Delta_{x} \otimes i d\right) R(x)=R_{13}\left(x q^{H_{2}}\right) R_{23}(x) F_{12}^{-1}\left(x q^{H_{3}}\right) F_{12}(x) \\
& \left(i d \otimes \Delta_{x}\right) R(x)=F_{23}^{-1}(x) F_{23}\left(x q^{H_{1}}\right) R_{13}(x) R_{12}\left(x q^{H_{3}}\right)
\end{aligned}
$$

Notice that for $x=0,\left.F_{12}(x)\right|_{x=0}=1$ and therefore $\left.R(x)\right|_{x=0}=R^{D}$. In the limit $x=\infty,\left.F_{12}^{-1}(x)\right|_{x=\infty}=q^{-H \otimes H / 2} R_{12}^{D}$. Thus $\left.R_{12}(x)\right|_{x=\infty}=q^{-H \otimes H / 2} R_{21}^{D} q^{H \otimes H / 2}$, and $\Delta_{x=\infty}(a)=$ $q^{-H \otimes H / 2} \Delta^{\prime}(a) q^{H \otimes H / 2}$ for all $a \in U_{q}\left(s l_{2}\right)$.

\section{Relation to 3 -j and 6-j symbols}

We now give a list of formulae expressing the matrix elements of the various objects we have considered so far in terms of standard q-analogs of the 3-j and 6-j symbols. Let $\rho^{(j)}$ denote the spin $\mathrm{j}$ representation of $\mathcal{U}_{q}\left(s l_{2}\right)$. Then

$$
\begin{aligned}
\rho^{(j)}(H)|j, m\rangle & =2 m|j, m\rangle \\
\rho^{(j)}\left(E_{ \pm}\right)|j, m\rangle & =\sqrt{[j \mp m][j \pm m+1]}|j, m \pm 1\rangle
\end{aligned}
$$

The first step is to find the matrix elements of the matrix $M(x)$ in the spin-j representation. We get

$$
\begin{aligned}
{\left[M^{\left(j_{1}\right)}(x)\right]_{\sigma_{1} m_{1}}=} & (-1)^{\sigma_{1}+m_{1}} \frac{\sqrt{\left[j_{1}+\sigma_{1}\right] !\left[j_{1}-\sigma_{1}\right] !\left[j_{1}+m_{1}\right] !\left[j_{1}-m_{1}\right] !}}{\prod_{r=1}^{j_{1}+\sigma_{1}}\left(1-x^{2} q^{2 r}\right)} . \\
& \cdot q^{\sigma_{1}\left(\sigma_{1}-m_{1}\right)} x^{\sigma_{1}-m_{1}} \sum_{p} \frac{q^{2 p \sigma_{1}} x^{2 p}}{[p] !\left[\sigma_{1}-m_{1}+p\right] !\left[j_{1}-\sigma_{1}-p\right] !\left[j_{1}+m_{1}-p\right] !}
\end{aligned}
$$


This formula agrees (up to normalizations) with the one found in [10].

This matrix $M(x)$ is known to perform the vertex-IRF transformation in conformal field theory [6, 6, 8, 9].

$$
\xi_{m_{1}}^{\left(j_{1}\right)}(z)=\sum_{\sigma_{1}} \psi_{\sigma_{1}}^{\left(j_{1}\right)}(z) M_{\sigma_{1} m_{1}}^{\left(j_{1}\right)}(x)
$$

where the $\psi$ 's are IRF type operators and the $\xi$ 's are vertex type operators. The braiding relations of the $\psi$ 's are described by the matrix $R(x)$, while those of the $\xi$ 's are described by $R^{D}$. Thus, we expect the elements $M_{\sigma_{1} m_{1}}^{\left(j_{1}\right)}(x)$ to be related to quantum 3 -j symbols. The precise connexion was found in [1]. We have

$$
\left[M^{\left(j_{1}\right)}(x)\right]_{\sigma_{1} m_{1}}=\frac{\mathcal{N}_{\psi}^{\left(j_{1}\right)}\left(x, \sigma_{1}\right)}{\mathcal{N}_{\xi}^{\left(j_{1}\right)}\left(m_{1}\right)} \lim _{m \rightarrow \infty}\left[\begin{array}{ccc}
j_{1} & j(x) & j(x)+\sigma_{1} \\
m_{1} & m & m+m_{1}
\end{array}\right]_{q}
$$

where we have defined $j(x)$ through the relation

$$
x=q^{2 j(x)+1}
$$

Eq.(14) has to be understood as an analytic continuation in $j(x)$ of 3 -j symbols [12]. We give a sketch of the proof in the Appendix. The factors $\mathcal{N}_{\xi}^{\left(j_{1}\right)}$ and $\mathcal{N}_{\psi}^{\left(j_{1}\right)}$ can be reabsorbed into the normalizations of the fields $\psi$ and $\xi$ respectively. Their expression is also given in the Appendix. We represent eq.(14) by a diagram

$$
\left[M^{\left(j_{1}\right)}(x)\right]_{\sigma_{1} m_{1}}=\begin{array}{l|l}
j(x)+\sigma_{1} & j(x) \\
\hline
\end{array}
$$

From eq.(14), it is now possible to build the complete dictionary between the matrix elements of $F_{12}(x)$ and $R_{12}(x)$ and standard 3 -j and 6 -j symbols.

We start with

$$
\begin{aligned}
& \left\langle j_{1}, \sigma_{1}\left|\left\langle j_{2}, \sigma_{2}\left|M_{2}^{\left(j_{2}\right)}\left(x q^{H_{1}}\right) M_{1}^{\left(j_{1}\right)}(x)\right| j_{1}, m_{1}\right\rangle\right| j_{2}, m_{2}\right\rangle=M_{\sigma_{2}, m_{2}}^{\left(j_{2}\right)}\left(x q^{2 \sigma_{1}}\right) M_{\sigma_{1}, m_{1}}^{\left(j_{1}\right)}(x)= \\
& \frac{\mathcal{N}_{\psi}^{\left(j_{1}\right)}\left(x, \sigma_{1}\right) \mathcal{N}_{\psi}^{\left(j_{2}\right)}\left(x q^{2 \sigma_{1}}, \sigma_{2}\right)}{\mathcal{N}_{\xi}^{\left(j_{1}\right)}\left(m_{1}\right) \mathcal{N}_{\xi}^{\left(j_{2}\right)}\left(m_{2}\right)} \lim _{m, m^{\prime} \rightarrow \infty}\left[\begin{array}{ccc}
j_{2} & j(x)+\sigma_{1} & j(x)+\sigma_{1}+\sigma_{2} \\
m_{2} & m & m+m_{2}
\end{array}\right]\left[\begin{array}{ccc}
j_{1} & j(x) & j(x)+\sigma_{1} \\
m_{1} & m^{\prime} & m^{\prime}+m_{1}
\end{array}\right]
\end{aligned}
$$

Notice that we have used the fact that

$$
x q^{2 \sigma_{1}}=q^{2\left(j(x)+\sigma_{1}\right)+1}
$$

Hence the shift in the Gervais-Neveu-Felder equation $x \rightarrow x q^{H}$ precisely corresponds to the shift of spins $j(x) \rightarrow j(x)+\sigma$. Thus we have the diagramatic correspondance

$$
M_{2}^{\left(j_{2}\right)}\left(x q^{H_{1}}\right) M_{1}^{\left(j_{1}\right)}(x)=\begin{array}{r|l|l}
j_{2} & j_{1} \\
\hline
\end{array}
$$


The matrix elements of $R_{12}(x)$ are computed from the formula

$$
R_{12}(x) M_{1}\left(x q^{H_{2}}\right) M_{2}(x)=M_{2}\left(x q^{H_{1}}\right) M_{1}(x) R_{12}^{D}
$$

or graphically

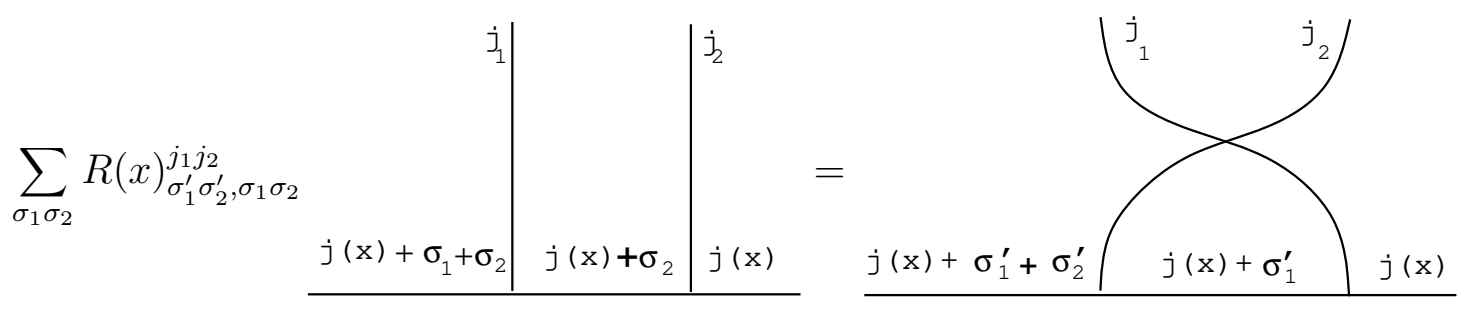

This is equivalent to the braiding relation and relates the matrix elements of $R(x)$ to 6-j symbols:

$$
\begin{gathered}
\left\langle j_{1}, \sigma_{1}^{\prime}\left|\left\langle j_{2}, \sigma_{2}^{\prime}\left|R_{12}(x)\right| j_{1}, \sigma_{1}\right\rangle\right| j_{2}, \sigma_{2}\right\rangle=(-1)^{\sigma_{1}^{\prime}-\sigma_{1}} q^{C(j(x))+C\left(j(x)+\sigma_{1}+\sigma_{2}\right)-C\left(j(x)+\sigma_{1}^{\prime}\right)-C\left(j(x)+\sigma_{2}\right)} \\
\frac{\mathcal{N}_{\psi}^{\left(j_{1}\right)}\left(x, \sigma_{1}^{\prime}\right) \mathcal{N}_{\psi}^{\left(j_{2}\right)}\left(x q^{2 \sigma_{1}^{\prime}}, \sigma_{2}^{\prime}\right)}{\mathcal{N}_{\psi}^{\left(j_{1}\right)}\left(x q^{2 \sigma_{2}}, \sigma_{1}\right) \mathcal{N}_{\psi}^{\left(j_{2}\right)}\left(x, \sigma_{2}\right)}\left\{\begin{array}{ccc}
j_{2} & j(x)+\sigma_{1}+\sigma_{2} & j(x)+\sigma_{1}^{\prime} \\
j_{1} & j(x) & j(x)+\sigma_{2}
\end{array}\right\}_{q}
\end{gathered}
$$

where $C(j)=j(j+1)$ and the last symbol represents a 6 -j coefficient (see eq. 5.11 in [13]).

Finally, we give the formula for the matrix elements of $F_{12}(x)$ in terms of $3-\mathrm{j}$ and $6-\mathrm{j}$ symbols. We start from the formula

$$
F_{12}(x) M_{1}\left(x q^{H_{2}}\right) M_{2}(x)=\Delta M(x)
$$

From the definition of the coproduct, we have

$$
\left[\Delta M^{j_{1} j_{2}}(x)\right]_{\sigma_{1} \sigma_{2}, m_{1} m_{2}}=\sum_{j_{12}}\left[\begin{array}{ccc}
j_{1} & j_{2} & j_{12} \\
\sigma_{1} & \sigma_{2} & \sigma_{1}+\sigma_{2}
\end{array}\right]_{q}\left[M^{\left(j_{12}\right)}(x)\right]_{\sigma_{1}+\sigma_{2}, m_{1}+m_{2}}\left[\begin{array}{ccc}
j_{1} & j_{2} & j_{12} \\
m_{1} & m_{2} & m_{1}+m_{2}
\end{array}\right]_{q}
$$

Using the interpretation of $M$ as a 3-j symbol and the defining relation of 6-j symbols, we can write

$$
\begin{aligned}
& {\left[M^{\left(j_{12}\right)}(x)\right]_{\sigma_{1}+\sigma_{2}, m_{1}+m_{2}}\left[\begin{array}{ccc}
j_{1} & j_{2} & j_{12} \\
m_{1} & m_{2} & m_{1}+m_{2}
\end{array}\right]_{q}=} \\
& \sum_{\sigma_{1}^{\prime} \sigma_{2}^{\prime}} \frac{\mathcal{N}_{\psi}^{\left(j_{12}\right)}\left(x, \sigma_{1}+\sigma_{2}\right)}{\mathcal{N}_{\psi}^{\left(j_{1}\right)}\left(x q^{2 \sigma_{2}^{\prime}}, \sigma_{1}^{\prime}\right) \mathcal{N}_{\psi}^{\left(j_{2}\right)}\left(x, \sigma_{2}^{\prime}\right)}\left\{\begin{array}{ccc}
j_{1} & j_{2} & j_{12} \\
j(x) & j(x)+\sigma_{1}+\sigma_{2} & j(x)+\sigma_{2}^{\prime}
\end{array}\right\}_{q} M_{\sigma_{1}^{\prime}, m_{1}}^{\left(j_{1}\right)}\left(x q^{2 \sigma_{2}^{\prime}}\right) M_{\sigma_{2}^{\prime}, m_{2}}^{\left(j_{2}\right)}(x)
\end{aligned}
$$

Hence

$$
\begin{gathered}
{\left[F^{j_{1} j_{2}}(x)\right]_{\sigma_{1} \sigma_{2}, \sigma_{1}^{\prime} \sigma_{2}^{\prime}}=} \\
\sum_{j_{12}} \frac{\mathcal{N}_{\psi}^{\left(j_{12}\right)}\left(x, \sigma_{1}+\sigma_{2}\right)}{\mathcal{N}_{\psi}^{\left(j_{1}\right)}\left(x q^{2 \sigma_{2}^{\prime}}, \sigma_{1}^{\prime}\right) \mathcal{N}_{\psi}^{\left(j_{2}\right)}\left(x, \sigma_{2}^{\prime}\right)}\left[\begin{array}{ccc}
j_{1} & j_{2} & j_{12} \\
\sigma_{1} & \sigma_{2} & \sigma_{1}+\sigma_{2}
\end{array}\right]_{q}\left\{\begin{array}{ccc}
j_{1} & j_{2} & j_{12} \\
j(x) & j(x)+\sigma_{1}^{\prime}+\sigma_{2}^{\prime} & j(x)+\sigma_{2}^{\prime}
\end{array}\right\}_{q}
\end{gathered}
$$




\section{Application to the trigonometric q-deformed Lamé equation}

In [3] it was shown how solutions of eq.(11) could be used to construct a set of commuting operators corresponding to $q$-deformations of the quantum Calogero-Moser Hamiltonians. In the $\mathcal{U}_{q}\left(s l_{2}\right)$ case, there is only one such operator once we separate the center of mass motion.

According to the general prescription [3], we start from a Lax matrix satisfying

$$
R_{12}\left(x q^{-\frac{1}{2} H_{3}}\right) L_{13}(x) L_{23}(x)=L_{23}(x) L_{13}(x) R_{12}\left(x q^{\frac{1}{2} H_{3}}\right)
$$

with a subscript 3 denoting the quantum space. The first Hamiltonian is the restriction of $\operatorname{Tr}_{1}\left(L_{13}(x)\right)$ to the subspace of zero-weight vectors.

In the representation $\rho=\rho^{(1 / 2)} \otimes \rho^{(1 / 2)}$ of $\mathcal{U}_{q}\left(s l_{2}\right) \otimes \mathcal{U}_{q}\left(s l_{2}\right)$, the following extra property is true:

$$
\rho\left(\left[\left(H_{1}+H_{2}\right) \partial_{x}, R_{12}(x)\right]\right)=0 .
$$

This condition allows to recast eq.(11) in the form (19) with a Lax operator $L(x)$ obtained by dressing $R(x)$ with suitable shift operators:

$$
L_{13}(x)=q^{-\left(H_{1}+\frac{1}{2} H_{3}\right) p} R_{13}(x) q^{\frac{1}{2} H_{3} p}, \quad \text { with } \quad p=x \frac{\partial}{\partial x} .
$$

In the representation $\rho_{j}=\rho^{(1 / 2)} \otimes \rho^{(j)}$ of $\mathcal{U}_{q}\left(s l_{2}\right) \otimes \mathcal{U}_{q}\left(s l_{2}\right)$,

$$
\rho_{j}\left(L_{13}(x)\right)=\left(\begin{array}{cc}
q^{-p} q^{\frac{1}{2} H} & -q^{-\frac{1}{2}} x^{-1} f\left(x q^{\frac{1}{2} H}\right) q^{-\frac{1}{2} H} E_{-} \\
q^{-\frac{1}{2}} x f\left(x q^{-\frac{1}{2} H+1}\right) q^{\frac{1}{2} H} E_{+} & q^{p} q^{-\frac{1}{2} H}\left[1-f\left(x q^{-\frac{1}{2} H}\right) f\left(x q^{\frac{1}{2} H-1}\right) E_{+} E_{-}\right]
\end{array}\right)
$$

with $f(x)=\left(q-q^{-1}\right) /\left(x-x^{-1}\right)$.

Taking the trace on the first space we get

$$
\operatorname{Tr}_{1}\left(L_{13}(x)\right)=q^{-p} q^{\frac{1}{2} H}+q^{p} q^{-\frac{1}{2} H}\left[1-f\left(x q^{-\frac{1}{2} H}\right) f\left(x q^{\frac{1}{2} H-1}\right) E_{+} E_{-}\right] .
$$

We still have to restrict this operator to the space of zero-weight vectors. In the spin $j$, representation, when $j$ is integer, this subspace is one-dimensional and the resulting Hamiltonian is scalar. Using $E_{+} E_{-}|j, 0\rangle=[j][j+1]|j, 0\rangle$, we get

$$
H_{j}=q^{-p}+q^{p}\left(1-\frac{\left(q-q^{-1}\right)^{2}[j][j+1]}{\left(x-x^{-1}\right)\left(q^{-1} x-q x^{-1}\right)}\right) .
$$

At the first non-trivial order of $H_{j}$ in the limit $q \rightarrow 1$, we recover the Calogero-Moser Hamiltonian $-\partial_{z}^{2}+j(j+1) / \sinh ^{2}(z)$, with $x=\exp (z)$. Notice that the coupling constant is related to the spin of the representation.

Alternatively, introducing the function

$$
c_{j}(x)=\frac{\left(q^{j} x-q^{-j} x^{-1}\right)\left(q^{-j-1} x-q^{j+1} x^{-1}\right)}{\left(x-x^{-1}\right)\left(q^{-1} x-q x^{-1}\right)},
$$

the Hamiltonian $H_{j}$ is given by

$$
H_{j}=q^{-p}+q^{p} c_{j}(x)
$$


The eigenfunctions $\Psi$ of $H_{j}$ are the solutions of the following trigonometric $q$-deformed Lamé equation:

$$
\Psi\left(q^{-1} x\right)+c_{j}(q x) \Psi(q x)=E \Psi(x) .
$$

An elliptic version of this equation already appeared in a different context in [14].

Integrability of the system manifests itself in the fact that we can easily solve this equation by using, for instance, the following recursive procedure. For $j=0$ the Hamiltonian $H_{0}=$ $q^{p}+q^{-p}$ is free; its eigenfunctions are plane waves $\Psi_{0}(x, k)=x^{k}$ with the corresponding energy $E(k)=q^{k}+q^{-k}$. Let us now introduce the following "shift" operator

$$
D_{j}=q^{-p}-q^{p} \frac{\left(q^{-j} x-q^{j} x^{-1}\right)\left(q^{-j-1} x-q^{j+1} x^{-1}\right)}{\left(x-x^{-1}\right)\left(q^{-1} x-q x^{-1}\right)}
$$

which satisfies

$$
H_{j} D_{j}=D_{j} H_{j-1},
$$

The eigenfunction $\Psi_{j}(x, k)$ of $H_{j}$ with energy $E(k)=q^{k}+q^{-k}$, are obtained by the successive action of the "shift" operator:

$$
\Psi_{j}(x, k)=D_{j} \Psi_{j-1}(x, k) .
$$

Since the energy is even in $k$, we can start the recursion with $\Psi_{0}(x, k)=x^{k}-x^{-k}$. Then we get

$$
\Psi_{j}(x, k)=\sum_{n=0}^{j}(-1)^{n}\left[\begin{array}{l}
j \\
n
\end{array}\right]_{q} \frac{\prod_{r=1}^{n}\left(q^{r-j-1} x-q^{-r+j+1} x^{-1}\right)}{\prod_{r=1}^{n}\left(q^{r} x-q^{-r} x^{-1}\right)}\left(q^{k(2 n-j)} x^{k}-q^{-k(2 n-j)} x^{-k}\right)
$$

This wave function has the interesting properties that the residues at the poles $x= \pm q^{-r}$ for $1 \leq r \leq j$ all vanish, and moreover, one has $\Psi_{j}(x, k)=0$ for $k=-j,-j+1, \cdots, j$. This is an analogue of the generalized exclusion principle present in the Calogero-Sutherland model [15.

\section{Appendix}

We give an idea of the proof of eq.(14). We adopt here a naive point of vue. We refer to [11] for a more detailed discussion. We start from an avatar of van der Waerden formula for $3-\mathrm{j}$ symbols (combine eq. 3.5 and eq. 3.10 in ref.[13]):

$$
\begin{aligned}
& {\left[\begin{array}{ccc}
j_{1} & j_{2} & j_{3} \\
m_{1} & m_{2} & m_{3}
\end{array}\right]_{q}=\delta_{m_{1}+m_{2}, m_{3} \Delta\left(j_{1}, j_{2}, j_{3}\right) q^{-\frac{1}{2}\left(j_{1}+j_{2}-j_{3}\right)\left(j_{1}+j_{2}+j_{3}+1\right)+j_{1} m_{2}-j_{2} m_{1}} \sqrt{\left[2 j_{3}+1\right]}} \cdot} \\
& \cdot \sqrt{\left[j_{1}+m_{1}\right] !\left[j_{1}-m_{1}\right] !\left[j_{2}+m_{2}\right] !\left[j_{2}-m_{2}\right] !\left[j_{3}+m_{3}\right] !\left[j_{3}-m_{3}\right] !} \cdot \\
& \quad \sum_{p} \frac{(-1)^{p} q^{p\left(j_{1}+j_{2}+j_{3}+1\right)}}{[p] !\left[j_{1}+j_{2}-j_{3}-p\right] !\left[j_{2}-m_{2}-p\right] !\left[j_{1}+m_{1}-p\right] !\left[j_{3}-j_{1}+m_{2}+p\right] !\left[j_{3}-j_{2}-m_{1}+p\right] !}
\end{aligned}
$$

where

$$
\Delta\left(j_{1}, j_{2}, j_{3}\right)=(-1)^{j_{1}+j_{2}-j_{3}} \sqrt{\frac{\left[-j_{1}+j_{2}+j_{3}\right] !\left[j_{1}-j_{2}+j_{3}\right] !\left[j_{1}+j_{2}-j_{3}\right] !}{\left[j_{1}+j_{2}+j_{3}+1\right] !}}
$$


We take a limit $m_{2} \rightarrow \infty$ such that

$$
\lim _{m_{2} \rightarrow \infty} q^{m_{2}}=0, \quad \lim _{m_{2} \rightarrow \infty} q^{-m_{2}}=\infty
$$

Then, one has

$$
\frac{\left[\alpha \pm m_{2}\right] !}{\left[\beta \pm m_{2}\right] !} \sim(\mp)^{\alpha-\beta} \frac{q^{\mp \frac{1}{2}(\alpha-\beta)(\alpha+\beta+1)}}{\left(q-q^{-1}\right)^{\alpha-\beta}} q^{-(\alpha-\beta) m_{2}}
$$

To perform the limit, we write the terms containing $m_{2}$ in the following form

$$
\begin{gathered}
\sqrt{\frac{\left[j_{2}+m_{2}\right] !}{\left[j_{3}-j_{1}+m_{2}\right] !} \cdot \frac{\left[j_{3}+m_{1}+m_{2}\right] !}{\left[j_{3}-j_{1}+m_{2}\right] !} \cdot \frac{\left[j_{2}-m_{2}\right] !}{\left[j_{2}-m_{2}\right] !} \cdot \frac{\left[j_{3}-m_{1}-m_{2}\right] !}{\left[j_{2}-m_{2}\right] !}} \\
(-1)^{j_{1}+\frac{1}{2}\left(j_{2}-j_{3}+m_{1}\right)} \frac{q^{\frac{1}{2}\left(-2 j_{3} m_{1}-m_{1}-2 j_{1}\left(j_{3}+1\right)+j_{1}\left(j_{1}+1\right)+j_{3}\left(j_{3}+1\right)-j_{2}\left(j_{2}+1\right)\right)}}{\left(q-q^{-1}\right)^{j_{1}}} q^{-j_{1} m_{2}}
\end{gathered}
$$

and

$$
\lim _{m_{2} \rightarrow \infty} \frac{\left[j_{2}-m_{2}\right] !\left[j_{3}-j_{1}+m_{2}\right] !}{\left[j_{2}-m_{2}-p\right] !\left[j_{3}-j_{1}+m_{2}+p\right] !}=(-1)^{p} q^{p\left(j_{2}+j_{3}-j_{1}+1\right)}
$$

This decomposition is to ensure that we get the above important sign $(-1)^{p}$ correctly. Hence

$$
\begin{aligned}
& \lim _{m_{2} \rightarrow \infty}\left[\begin{array}{ccc}
j_{1} & j_{2} & j_{3} \\
m_{1} & m_{2} & m_{1}+m_{2}
\end{array}\right]_{q}= \Delta\left(j_{1}, j_{2}, j_{3}\right) \frac{\sqrt{\left[2 j_{3}+1\right]} \sqrt{\left[j_{1}+m_{1}\right] !\left[j_{1}-m_{1}\right] !}}{\left(q-q^{-1}\right)^{j_{1}}} \cdot \\
& \cdot(-1)^{j_{1}+\frac{1}{2}\left(j_{2}-j_{3}+m_{1}\right)} q^{-j_{2}\left(j_{2}+1\right)+j_{3}\left(j_{3}+1\right)-j_{1}\left(j_{3}+j_{2}+1\right)} q^{-\frac{1}{2} m_{1}} \cdot \\
& \cdot q^{-\left(j_{2}+j_{3}\right) m_{1}} \sum_{p} \frac{q^{2 p\left(j_{2}+j_{3}+1\right)}}{[p] !\left[j_{1}+j_{2}-j_{3}-p\right] !\left[j_{1}+m_{1}-p\right] !\left[j_{3}-j_{2}-m_{1}+p\right] !}
\end{aligned}
$$

Comparing with eq.(13) we get eq.(14) with $j_{2}=j(x)$ and $j_{3}=j(x)+\sigma_{1}$ where $j(x)$ is given by eq.(15). Moreover we find

$$
\begin{aligned}
\mathcal{N}_{\xi}^{\left(j_{1}\right)}\left(m_{1}\right) & =(-1)^{-\frac{1}{2} m_{1}} q^{\frac{1}{2} m_{1}} \\
\mathcal{N}_{\psi}^{\left(j_{1}\right)}\left(x, \sigma_{1}\right) & =(-1)^{-j_{1}+\frac{1}{2}\left(m_{1}-\sigma_{1}\right)} \frac{\sqrt{\left[j_{1}+\sigma_{1}\right] !\left[j_{1}-\sigma_{1}\right] !}}{\prod_{r=1}^{j_{1}+\sigma_{1}}\left(1-x^{2} q^{2 r}\right)} \frac{\left(q-q^{-1}\right)^{j_{1}} x^{j_{1}} q^{j_{1} \sigma_{1}}}{\Delta\left(j_{1}, j(x), j(x)+\sigma_{1}\right) \sqrt{\left[2 j(x)+1+\sigma_{1}\right]}}
\end{aligned}
$$

\section{References}

[1] J.L. Gervais, A. Neveu, Novel triangle relation and absence of tachyons in Liouville string field theory, Nucl. Phys. B 238 (1984) 125.

[2] G. Felder, Conformal field theory and integrable systems associated to elliptic curves, hep-th/9407154. 
[3] J. Avan, O. Babelon, E. Billey The Gervais-Neveu-Felder equation and the quantum Calogero Moser systems. hep/th 9505091, To appear in Commun. Math. Phys.

[4] O. Babelon, Universal exchange algebra for Bloch waves and Liouville theory, Commun. Math. Phys. 139 (1991) 619.

[5] V.G. Drinfeld, Quasi-Hopf algebras. Algebra and Analysis, 1 (1989) p. 1419. On quasitriangular quasi-Hopf algebras and a group closely connected with $\operatorname{Gal}(\bar{Q} / Q)$. Algebra and Analysis, 2 (1990) p.829.

[6] V. Pasquier, Etiology of IRF models. Commun. Math. Phys. 118 (1988) p.355.

[7] O. Babelon, Extended conformal algebra and the Yang-Baxter equation, Phys. Lett. B 215 (1988) 523.

[8] G. Moore, N. Reshetikhin, A comment on quantum group symmetry in conformal field theory. Nucl. Phys. B328 (1989) p. 557.

[9] G. Felder, J. Fröhlich, J, Keller, Braid matrices and structure constants for minimal conformal models. Commun. Math. Phys. 124 (1989) p. 646.

[10] J.L. Gervais, The Quantum Group Structure of $2 D$ Gravity and Minimal Models, Commun Math Phys 130, (1990) p. 257.

[11] E. Cremmer, J.L. Gervais, J.F. Roussel, The Quantum Group Structure of 2D Gravity and Minimal Models II: The Genus-Zero Chiral Bootstrap. Commun. Math. Phys. 161, (1994), p. 597.

[12] J.L. Gervais, J.F. Roussel, Solving the strongly coupled 2D gravity II. Fractional-spin operators and topological three point functions. Nucl. Phys. B426 (1994) p.140.

[13] A.N. Kirillov, N.Yu. Reshetikhin, Representations of the algebra $\mathcal{U}_{q}\left(s_{2}\right)$, q-orthogonal polynomials and invariants of links. Infinite dimensional Lie algebras and groups. Advanced Study in Mathematical Physics, Vol 7, Proceedings of the 1988 Marseille Conference. World Scientific.

[14] I. Krichever, A. Zabrodin, Spin generalization of the Ruijsenaars-Schneider model, nonabelian 2D Toda chain and representations of Sklyanin algebra, hep-th/9505039.

[15] D. Bernard, M. Gaudin, F. Haldane, V. Pasquier, Yang-Baxter equation in spin chains with long range interactions. J. Phys. A: Math. Gen. 26 (1993) p.5219. 